\title{
Modelamiento sísmico en base a unidades de albañilería de suelo cemento en la región Amazonas
}

\section{Seismic modeling based on concrete floor masonry units, Amazonas region}

\author{
Walter Ramírez Vásquez
}

\section{RESUMEN}

La presente investigación tuvo como objetivo determinar la respuesta al modelamiento sísmico de una vivienda en base a unidades de albañilería suelo cemento, en la Villa San Juan, Magdalena, región Amazonas, Perú, para tal finalidad se hizo la extracción de muestras de suelo y arena, para el análisis correspondiente en el Laboratorio de Suelos, Concreto y Asfalto de la Dirección Regional de Transportes y Comunicaciones de la región Amazonas, de ello se obtuvo una mezcla, con $40 \%$ de aporte de tierra colpar y un $60 \%$ de aporte arena de cerro, procediendo a elaborar el Ladrillo de Tierra Comprimida, con adición de 7\%, 11\%, 15\% y 19\% de cemento en peso respecto a la mezcla, determinándose sus propiedades físico-mecánicas; lo que generó estadísticamente un mejor comportamiento en sus propiedades físicas y mecánicas, en el lote experimental $\mathrm{L}_{3}(19 \%)$, concluyéndose que el modelamiento sísmico de la vivienda modelo, presenta respuesta admisible según normas E 0.70, E 0.30, E0.20 del RNE.

\section{Palabras clave: Modelamiento Sísmico, Ladrillo de Tierra}

\begin{abstract}
The objective of this research was to determine the response to the seismic modeling of a house based on cementsoil masonry units, in Villa San Juan, Magdalena, Amazonas región, Peru, for this purpose the extraction of soil and sand samples was carried out, for their corresponding analysis in the Laboratory of Soils, Concrete and Asphalt of the Regional Directorate of Transport and Communications, Amazon region, from which a mixture was obtained, with $40 \%$ contribution of colpar earth and $60 \%$ contribution of hill sand, proceeding to elaborate Compressed Earth Brick, with the addition of $7 \%, 11 \%, 15 \%$ and $19 \%$ of cement by weight with respect to the mixture, determining its physical-mechanical properties; which statistically generated a better performance in its physical and mechanical properties, in the experimental lot $\mathrm{L}_{3}(19 \%)$, concluding that the seismic modeling of the model house, presents an admissible response according to norms E 0.70, E 0.30, E0.20 of the RNE.
\end{abstract}

Keywords: Seismic Modeling, Earth Brick

${ }^{1}$ Escuela Profesional de Ingeniería Civil. Universidad Nacional Toribio Rodríguez de Mendoza de Amazonas. Correo electrónico: ramirez_wvz2@hotmail.com 


\section{INTRODUCCIÓN}

En una práctica supervisada por la Universidad Nacional de Córdoba en Argentina, se elaboró bloques de suelo cemento con aporte de rediseño de cemento del $11 \%$ de cemento a la dosificación $40 \%$ de suelo con $60 \%$ de arena, obteniendo una resistencia a la compresión promedio de $5.82 \mathrm{Mpa}=$ $59.40 \mathrm{~kg} / \mathrm{cm}^{2}$ admisible con sus parámetros (Berlingieri, 2017).

En la investigación realizada en Trujillo se realizó unidades de albañilería suelo cemento con aporte de cemento en $20 \%$ del peso del suelo, con características de suelo $75 \%$ de arena y $25 \%$ de limos y arcillas, resultando un $\mathrm{f}^{\prime} \mathrm{b}=74.78 \mathrm{~kg} / \mathrm{cm}^{2}$, un $\mathrm{f}^{\prime} \mathrm{m}=37.23 \mathrm{~kg} / \mathrm{cm}^{2}$ y un $\mathrm{V}^{\prime} \mathrm{m}=5.0 \mathrm{~kg} / \mathrm{cm}^{2}$ (Abanto $\mathrm{y}$ Akarley, 2014).

En la investigación realizada en Huancayo se elaboró ladrillos de tierra comprimida con aporte de cemento en $7 \%, 11 \%, 15 \%$ y $20 \%$ del peso del suelo, con características de suelo $48.60 \%$ de arena y $34.30 \%$ de limos y arcillas, resultando para la categoría LTC 7\% un $\mathrm{f}^{\prime} \mathrm{b}=23.12 \mathrm{~kg} / \mathrm{cm}^{2}$, inferior en $53.76 \%$ a la resistencia mínima requerida por la norma E0.70 (50 $\mathrm{kg} / \mathrm{cm}^{2}$ ) para ladrillos de Tipo I; para la categoría LTC $11 \%$ un $\mathrm{f}^{\prime} \mathrm{b}=27.99 \mathrm{~kg} / \mathrm{cm}^{2}$, inferior en $44 \%$ a la resistencia mínima requerida por la norma E0.70 (50 $\mathrm{kg} / \mathrm{cm}^{2}$ ) para ladrillos de Tipo I; para la categoría LTC $15 \%$ un $\mathrm{f}^{\prime} \mathrm{b}=52.29 \mathrm{~kg} / \mathrm{cm}^{2}$, superlativo en $4.58 \%$ a la resistencia mínima requerida por la norma E0.70 $\left(50 \mathrm{~kg} / \mathrm{cm}^{2}\right)$ para ladrillos de Tipo I; y para la categoría LTC $20 \%$ un $\mathrm{f}^{\prime} \mathrm{b}=69.09 \mathrm{~kg} / \mathrm{cm}^{2}$, superlativo en $38.18 \%$ a la resistencia mínima requerida por la norma E0.70 $\left(50 \mathrm{~kg} / \mathrm{cm}^{2}\right)$ para ladrillos de Tipo I (Meza, 2018).

Según el Instituto Geofísico del Perú (IGP), el 25 de Setiembre de 2005, se registró un terremoto de 7.0 grados de magnitud en la escala de Richter, con epicentro a 90 kilómetros al suroeste de la ciudad de Moyobamba, en la Región de San Martín, donde las viviendas más perjudicadas fueron las levantadas en base a tapial y adobe (Flores y Moromi, 2005).

En ese contexto, La villa San Juan, como anexo del Distrito de Magdalena, provincia Chachapoyas, no es ajeno al peligro sísmico, está expuesto a sufrir sismos de baja o alta intensidad, en la actualidad en respuesta a la actividad sísmica, encontramos viviendas construidas de adobe en mal estado con fallas en su estructura, lo cual esto ocasiona un grave problema para la seguridad de sus ocupantes.

La finalidad de esta investigación fue determinar la respuesta al modelamiento sísmico de una vivienda sobre la base de unidades de albañilería suelo cemento con aporte de cemento del 7\%, 11\%, 15\%,y $19 \%$ respecto al peso de la mezcla, así como caracterizar la mecánica de suelo donde se aplicó el modelo, elaborar la unidad de albañilería LTC y determinar sus propiedades Físico - Mecánicas y estadísticamente identificar el mejor lote, diseñar la arquitectura de la vivienda, así como también diseñar y analizar la estructuración de la vivienda de albañilería armada según el modelo utilizado.

\section{MATERIAL Y MÉTODO}

La investigación se realizó en el anexo Villa San Juan en el Distrito de Magdalena, provincia de Chachapoyas, región Amazonas, Perú.

El universo muestral fue 492 unidades de albañilería suelo cemento, fabricadas con Maquina Manual, en Jr. Sosiego Cuadra 7, Chachapoyas, región Amazonas.

Para desarrollar y validar esta investigación se estimó un muestreo no probabilístico, parametrizado a cada ensayo con 492 unidades de LTC (Meza, 2018).

Se inició de casos particulares en los estudios de muestras de suelos, y se adicionó cemento en 7\%, $11 \%, 15 \%$ y $19 \%$ respecto al peso de la mezcla $60 \%$ de arena de cerro y $40 \%$ de tierra colpar, para fabricar LTC, y conocer sus propiedades físicas y mecánicas sondeando estadísticamente el mejor lote, llegando a casos generales que se aplicaron en el modelamiento sísmico de una vivienda modelo.

En la extracción de tierra colpar, arena de cerro, arena del río Utcubamba, muestra de calicata C-1; se utilizó la observación directa; en el análisis de mezclas se utilizó el método gráfico, y en laboratorio se utilizó pruebas estandarizadas visadas por el laboratorio (Meza, 2018).

\section{Etapa 1: Estudio de Mecánica de Suelos.}

Fase Preliminar: Cantera tierra colpar se encuentra en Villa San Juan, cantera de arena de cerro está ubicada en el distrito de Magdalena, al costado derecho de la carretera a Magdalena, cantera de arena de río Utcubamba está ubicada en el distrito de Magdalena, provincia Chachapoyas, al costado izquierdo del río Utcubamba, y de estas se extrajo aproximadamente 50 kg de la muestra; calicata C-1 se seleccionó al azar un solar para la excavación de una calicata C-1, en Villa San Juan, excavándose $3 \mathrm{~m}$ de profundidad; y por último el laboratorio de la DRTC-AMAZONAS está ubicada en la dirección: km. $1+000$ carretera a Rodríguez de Mendoza, en la provincia de Chachapoyas.

Fase experimental: Se realizó los ensayos de mecánica de suelos para determinar las propiedades físicas y mecánicas del suelo, tanto de la tierra colpar, arena de cerro, arena de río y calicata $\mathrm{C}-1$, los cuales se hicieron mediante pruebas estandarizadas dadas por el MTC (Linares, 2019).

Fase de gabinete: Para la combinación de suelos aplico el método del cuadrado, se realiza asignando 
suelo A, a la arena de cerro y suelo B, a la tierra colpar, y a las especificaciones de granulometría dado por Toirac Corral (2008), obteniendo un 51.3\% para el suelo A, y un $48.7 \%$ para el suelo B, ajustándose en laboratorio, con las siguientes proporciones: $51 \%$ Arena de cerro - 49\% Tierra colpar, 55\% Arena de cerro - 45\% Tierra colpar, $60 \%$ Arena de cerro - 40\% Tierra colpar, 65\% Arena de cerro - 35\% Tierra colpar, y 70\% Arena de cerro $30 \%$ Tierra colpar.

\section{Etapa 2: Fabricación de Unidades de LTC}

Fase Preliminar: El taller de fabricación de LTC, está ubicado en Jirón Sosiego cuadra 7, Chachapoyas, Amazonas, el porcentaje de cemento según la PCA, para esta investigación de suelo A-2, se propuso el $7 \%$ de cemento, entonces los lotes experimentales se inicio por el $\mathrm{L}_{0}(7 \%)$, luego se aumentó el intervalo cada cuatro, siendo las adiciones al $\mathrm{L}_{0}(7 \%), \mathrm{L}_{1}(11 \%)$, $\mathrm{L}_{2}(15 \%), \mathrm{y}_{3}(19 \%)$ de cemento en peso respecto a la mezcla de arena cerro y tierra colpar.

Fase Experimental: Se moldeo unidades de LTC, usando prensa con palanca manual, poniendo a prueba de humedad optima, según Abanto Flores y Akarley Poma, 2014, luego se procedió al curado, secado y apilado de LTC, bajo sombra con cobertura de calamina, hasta completar los 28 días de llegar a su resistencia optima, para el armado de pilas y muretes.

Fase de gabinete: Diseño de dosificación para LTC, se midió la prensa con vernier, y se procedió a hacer el cálculo donde el volumen requerido es de 0.004 $\mathrm{m}^{3}$, con humedad de la mezcla insitu a tener en cuenta de $10.5 \%$, y con $5.22 \mathrm{~kg}$ de mezcla satisfació al volumen requerido, afectándose el aporte del $7 \%$, $11 \%, 15 \%$ y $19 \%$ para cemento, obteniéndose que para 492 LTC, se necesita para 7\%: arena cerro $(311.13 \mathrm{~kg})$, tierra colpar $(207.42 \mathrm{~kg})$, cemento $(36.2985 \mathrm{~kg})$, agua (58.259 lts); $11 \%$ : arena cerro $(306.475 \mathrm{~kg})$, tierra colpar $(204.317 \mathrm{~kg})$, cemento $(56.187 \mathrm{~kg})$, agua (59.53 lts); $15 \%$ : arena cerro $(301.957 \mathrm{~kg})$, tierra colpar $(201.305 \mathrm{~kg})$, cemento (75.489 kg), agua (60.769 lts); y 19\%: arena cerro $(297.57 \mathrm{~kg})$, tierra colpar $(198.38 \mathrm{~kg})$, cemento (94.23 kg), agua (61.969 lts); y para grout fino se hizo con cemento-arena: 1 -3, este diseño en gran medida está basado al diseño de mortero para pilas y muretes, hecho por Bach. Antonio Martin Tejada Arias, en la tesis: "Elaboración de un Ladrillo Alternativo sin Cocción en Cajamarca" (2013), cantidad de materiales en grout fino para llenado de alveolos de pilas y muretes de LTC.

se evaluó la cantidad total en volumen requerida en pilas y muretes, siendo $0.20855415 \mathrm{~m}^{3}$, con esto se procedió a sacar la cantidad total de cemento: $(456.1695)(0.20855415)=95.13604 \mathrm{~kg}$, total arena: $(1291.744)(0.20855415)=269.39857 \mathrm{~kg}$ y total Agua: $(364.9356)(0.20855415)=76.10884$ lts, de estos resultados totales, se puede observar que se necesita $95.13604 / 42.5=2.24$ bls de cemento.

\section{Etapa 3: Ensayos de Albañilería de Unidades de LTC}

Fase Preliminar: Se realizó en Laboratorio de suelos, concreto y asfalto de la DRTC-Amazonas, cabe mencionar un aporte de $46 \mathrm{LTC} / \mathrm{m}^{2}$.

Fase Experimental: Ensayos de albañilería se realizaron con la finalidad de determinar las propiedades físicas y mecánicas de la unidad de albañilería suelo cemento, los cuales se hicieron mediante pruebas estandarizadas dadas por la Norma Técnica Peruana.

Fase de Gabinete: Todos los lotes experimentales concerniente a variación dimensional, clasifican como ladrillo tipo $\mathrm{V}$, en cuanto a alabeo clasifican como ladrillo tipo $\mathrm{V}$ y en relación a la resistencia característica a la compresión solo en lote experimental de LTC 19\% llega a ser ladrillo tipo I, por otro lado tenemos un porcentaje de sección transversal: $\mathrm{L}_{0}(7 \%)$ de $80.68 \%, \mathrm{~L}_{1}(11 \%)$ de $80.26 \%$, $\mathrm{L}_{2}(15 \%)$ de $80.09 \%$, y $\mathrm{L}_{3}(19 \%)$ de $79.92 \%$, todos mayores al 70\% del área bruta (E.070-2006), por lo que clasifica como ladrillo alveolar sólido.

Además teniendo en cuenta que la dispersión de resultados en los ensayos de albañilería, ninguna muestra supero el $40 \%$ para unidades producidas artesanalmente (E.070-2006); conllevando que las unidades sean aceptables, para el caso de absorción la norma E.070-2006 plantea que no debe ser superior al $22 \%$, teniendo para $\mathrm{L}_{0}(7 \%)$ un porcentaje de absorción de $20.03 \%$, para $\mathrm{L}_{1}(11 \%)$ un porcentaje de absorción de $19.35 \%$, para $\mathrm{L}_{2}(15 \%)$ un porcentaje de absorción de $19.00 \%$, y para $\mathrm{L}_{3}(19 \%)$ un porcentaje de absorción de $18.59 \%$, por lo cual las unidades son aceptables de acuerdo a norma E.070-2006 (San Bartolomé, 2005).

\section{Etapa 4: Modelamiento Sísmico de una vivienda modelo}

Fase Preliminar: El modelo se aplicó a Villa San Juan del distrito de Magdalena, Chachapoyas, Amazonas, en un solar de $12 \mathrm{~m} \times 24 \mathrm{~m}$, de un piso con un área techada total de $264 \mathrm{~m}^{2}$, con una altura de $3.61 \mathrm{~m}$, incluyendo la cobertura y la altura de entrepiso es de $2.45 \mathrm{~m}$, teniendo un área neta de terreno de $200.80 \mathrm{~m}^{2}$.

Fase Experimental y de Gabinete: Características de la edificación modelo se ubica en la Villa San Juan, Magdalena, Chachapoyas, Amazonas, uso vivienda unifamiliar, sistema estructural albañilería armada, altura Total de la Edificación $3.61 \mathrm{~m}$, altura de muro piso a techo $\mathrm{h}=2.45 \mathrm{~m}$, altura de sobrecimiento sobresaliente de NPT $0.15 \mathrm{~m}$, altura libre de albañilería $\mathrm{h}=2.30 \mathrm{~m}$, espesor del bloque de LTC $\mathrm{t}=$ 
$0.125 \mathrm{~m}$, alfeizar $\mathrm{h}=0.90 \mathrm{~m}$ (excepto en SS.HH. donde $\mathrm{h}=1.75 \mathrm{~m}$ ), cobertura liviana (teja andina) con tijerales de madera, área techada cobertura 1er piso $264.00 \mathrm{~m}^{2}$, área de la planta típica muros 1er piso $176.0778 \mathrm{~m}^{2}$. A demás presento las características de los materiales: albañilería unidad de LTC, Grout fino $\mathrm{f}^{\prime} \mathrm{c}=140 \mathrm{~kg} / \mathrm{cm}^{2}$, f'b lote experimental $\mathrm{L}_{3} 52.81$ $\mathrm{kg} / \mathrm{cm}^{2}, \mathrm{f}^{\prime} \mathrm{m}$ lote experimental $\mathrm{L}_{3} 27.63 \mathrm{~kg} / \mathrm{cm}^{2}, \mathrm{~V}^{\prime} \mathrm{m}$ lote experimental $\mathrm{L}_{3} 5.62 \mathrm{~kg} / \mathrm{cm}^{2}, \mathrm{~V}^{\prime} \mathrm{m}$ de diseño 5.26 $\mathrm{kg} / \mathrm{cm}^{2}$, Em es $500 \mathrm{f}^{\prime} \mathrm{m}=13815 \mathrm{~kg} / \mathrm{cm}^{2}$, Gm es $0.4 \mathrm{Em}=5526 \mathrm{~kg} / \mathrm{cm}^{2}$, módulo de Poisson 0.25 , acero fy $=4200 \mathrm{~kg} / \mathrm{cm}^{2}$, teniendo en cuenta que el caso de las características del LTC, corresponde al lote experimental de $\mathrm{L}_{3}(19 \%)$, esto es por las pruebas múltiples de DMS, y para el V'm de diseño la norma E.070-2006 plantea que no debe ser mayor a 0.319 $\sqrt{f^{\prime} m} \mathrm{Mpa} \sqrt{f^{\prime} m} \mathrm{~kg} / \mathrm{cm}^{2}$ ), de donde $\sqrt{f^{\prime} m} \mathrm{~kg} / \mathrm{cm}^{2}=\sqrt{27.63}$

$\mathrm{kg} / \mathrm{cm}^{2}=5.26 \mathrm{~kg} / \mathrm{cm}^{2}$, este valor es menor al V'm = $5.62 \mathrm{~kg} / \mathrm{cm}^{2}$ del ensayo de laboratorio, por lo tanto, según norma E.070-2006 el valor del V'm de diseño será de $5.26 \mathrm{~kg} / \mathrm{cm}^{2}$.

\section{RESULTADOS}

Abreviaturas:

\begin{tabular}{|c|c|}
\hline $\begin{array}{l}\text { LL: Limite Liquido } \\
\text { LP: Límite Plástico } \\
\text { IP: Índice Plástico } \\
\text { SUCS: Sistema Unificado de Clasificación } \\
\text { de Suelos } \\
\text { ASSHTO: Asociación Americ ana de } \\
\text { Oficiales de Carreteras Estatales y } \\
\text { Transportes } \\
\text { E.A.: Equivalente de Arena } \\
\text { PUSS: Peso Unitario Suelto Seco en } \\
\text { kg/m3 } \\
\text { PUCS: Peso Unitario Comp. Seco en } \\
\text { kg/m3 } \\
\text { GEC: Gravedad Específica Corriente } \\
\text { GESSS: Gravedad Específica Saturada } \\
\text { Superficialmente Seca } \\
\text { GEA: Gravedad Específica Aparente } \\
\text { ABSR.: \% de Absorción } \\
\text { DMS: Densidad Máxima Seca } \\
\text { HO: Humedad Óptima } \\
\text { q adm: Tensión Admisible del Suelo } \\
\text { NP: No Presenta } \\
\text { OL: Limos orgánicos, arcillas limosas } \\
\text { orgánicas de baja plasticidad }\end{array}$ & 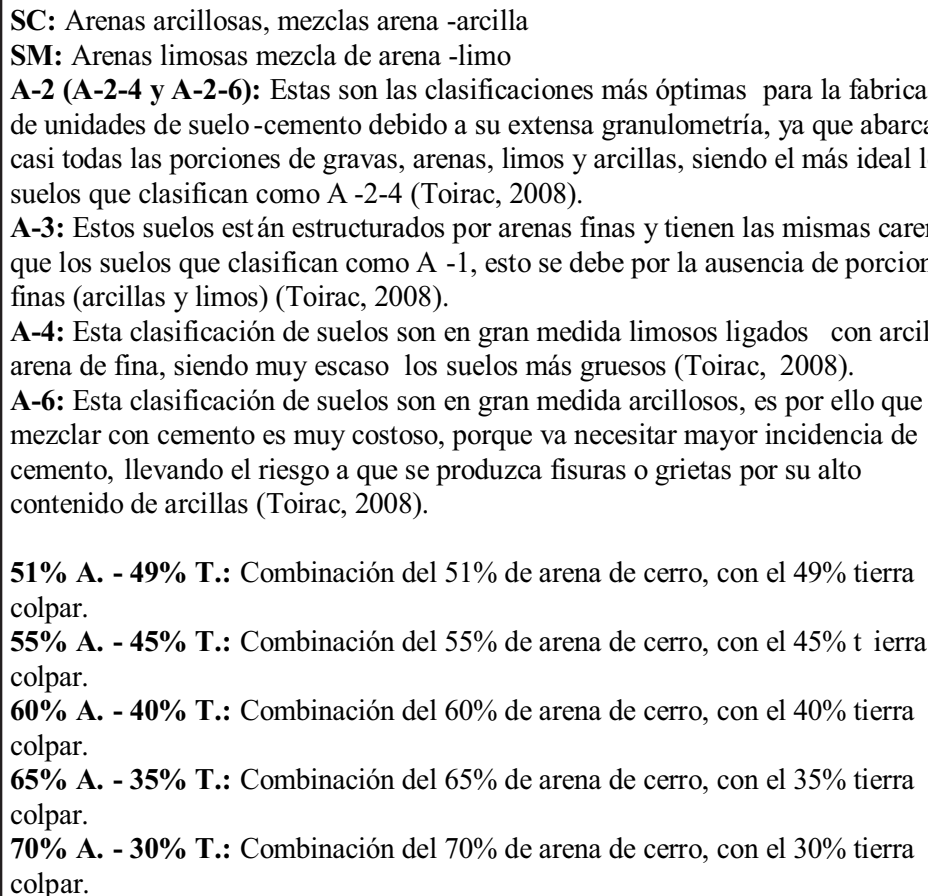 \\
\hline
\end{tabular}




\section{Tabla 1}

Resultados de ensayos de mecánica de suelos.

\begin{tabular}{|c|c|c|c|c|c|c|c|c|c|c|c|c|c|c|c|c|c|c|c|c|c|c|c|c|}
\hline \multirow[t]{2}{*}{ DESCRIPCIÓN } & \multicolumn{4}{|c|}{ GRANULO METRÍA } & \multicolumn{4}{|c|}{ CLASIFICACIÓN } & \multirow[t]{2}{*}{ HUM } & \multirow{2}{*}{$\begin{array}{c}\text { TAM } \\
\text { IZ } \\
200\end{array}$} & \multirow{2}{*}{$\begin{array}{l}\text { E. } \\
\text { A. }\end{array}$} & \multicolumn{2}{|c|}{$\begin{array}{c}\text { PESOS } \\
\text { UNITARIO } \\
\mathrm{S} \\
\end{array}$} & \multicolumn{4}{|c|}{ GRAV. ESP. Y ABSOR } & \multicolumn{2}{|c|}{$\begin{array}{c}\text { PROC. } \\
\text { ESTAND. }\end{array}$} & \multicolumn{2}{|c|}{$\begin{array}{c}\text { PROC. } \\
\text { INT. }\end{array}$} & \multirow{2}{*}{$\begin{array}{c}\text { PROC } \\
\text { MOD. } \\
\text { DMS }\end{array}$} & \multicolumn{2}{|c|}{$\begin{array}{c}\text { C. } \\
\text { DIREC }\end{array}$} \\
\hline & RAVA & ARE A & FINOS & LL & LP & IP & SUCS & AASHTO & & & & PUSS & PUCS & GEC & $\begin{array}{c}\text { GESS } \\
\mathrm{S}\end{array}$ & GEA & $\begin{array}{c}\text { ABS } \\
\mathbf{R}\end{array}$ & DMS & HO & DMS & HO & & HO & $\underset{\mathrm{adm}}{\mathbf{q}}$ \\
\hline COLPAR & 0.9 & 29.7 & 69.5 & 40 & 27 & 13 & $\mathrm{OL}$ & A-6(8) & 12.39 & 69.18 & 13 & 871.0 & 1090 & 1.691 & 2.037 & 2.586 & 20.488 & 1.277 & 17.7 & - & -- & - & - & - \\
\hline ARE. CERRO & 0.2 & 89.0 & 10.9 & 20 & 8 & 12 & SP-SC & A-2-6(0) & 6.08 & 10.43 & 28 & 1490.0 & 1729 & 2.344 & 2.422 & 2.541 & 3.306 & 1.815 & 12.0 & - & -- & -- & - & - \\
\hline CALICATA & 0.7 & 56.7 & 42.6 & 30 & 16 & 14 & $\mathrm{SC}$ & A-6(3) & 15.06 & - & - & - & 1311 & 2.345 & 2.406 & 2.498 & 2.627 & -- & - & - & - & - & - & 1.18 \\
\hline $51 \%$ A $-49 \%$ T. & 0.4 & 53.3 & 46.4 & 26 & 16 & 10 & $\mathrm{SC}$ & A-4 (2) & - & - & 18 & - & - & 2.470 & 2.499 & 2.544 & 1.174 & - & - & - & - & - & - & - \\
\hline $55 \% \mathrm{~A}-45 \% \mathrm{~T}$. & 0.2 & 63.0 & 36.8 & 23 & 13 & 10 & $\mathrm{SC}$ & $\mathrm{A}-4(0)$ & - & - & - & - & - & - & - & - & - & - & - & - & -- & - & - & - \\
\hline $60 \% \mathrm{~A}-40 \% \mathrm{~T}$. & 0.6 & 66.4 & 33.1 & 21 & 13 & 8 & $\mathrm{SC}$ & A-2-4(0) & - & - & : & 1217.1 & 1480 & 2.451 & 2.478 & 2.518 & 1.082 & 1.847 & 13.1 & 1.874 & 13.3 & 1.892 & 13.6 & - \\
\hline $65 \% \mathrm{~A}-35 \% \mathrm{~T}$. & 0.9 & 69.9 & 29.3 & 19 & 12 & 7 & $\mathrm{SC}$ & A-2-4 (0) & -- & - & - & - & - & - & - & - & -- & -- & -- & -- & -- & - & - & - \\
\hline $70 \% \mathrm{~A} .-30 \% \mathrm{~T}$. & 1.0 & 70.4 & 28.7 & 17 & 10 & 7 & $\mathrm{SC}$ & A-2-4 (0) & - & - & - & - & - & - & - & - & - & -- & - & - & - & - & - & - \\
\hline H. СAMPO & -- & - & - & - & - & - & - & -- & 3.00 & - & : & - & - & - & - & - & - & -- & - & - & - & - & - & - \\
\hline ARENA RIO & 11.1 & 80.5 & 8.4 & $\mathrm{NP}$ & $\mathrm{NP}$ & $\begin{array}{l}\mathrm{N} \\
\mathrm{P}\end{array}$ & $\begin{array}{l}\text { SP- } \\
\text { SM }\end{array}$ & $\mathrm{A}-3(0)$ & 3.28 & - & - & 1416.7 & 1668 & .604 & 2.639 & $\begin{array}{l}2.6 \\
97\end{array}$ & 1.317 & - & - & - & -- & -- & - & -- \\
\hline
\end{tabular}

Tabla 2

Resumen granulométrico de las mezclas de suelo.

\begin{tabular}{|c|c|c|c|c|c|c|}
\hline \multicolumn{2}{|c|}{ DESCRIPCIÓN } & \multirow{2}{*}{$\begin{array}{r}51 \% \text { A. } \\
49 \% \text { T.C } \\
0.4\end{array}$} & \multirow{2}{*}{$\begin{array}{c}\mathbf{5 5 \%} \% \text { A. } \\
\mathbf{4 5 \%} \text { T.C } \\
0.2\end{array}$} & \multirow{2}{*}{$\begin{array}{c}\text { 60\%A. } \\
\mathbf{4 0 \%} \text { T.C } \\
0.6\end{array}$} & \multirow{2}{*}{$\begin{array}{c}\mathbf{6 5 \%} \% \mathbf{A} \\
\mathbf{3 6} \% \text { T.C } \\
0.9\end{array}$} & \multirow{2}{*}{$\begin{array}{c}\mathbf{7 0} \% \mathbf{A} . \\
\mathbf{3 0 \%} \text { T.C } \\
1.0\end{array}$} \\
\hline Grava & Grava & & & & & \\
\hline Arena & Aren & 53.3 & 63.0 & 66.4 & 69.9 & 70.4 \\
\hline $\begin{array}{l}\text { Limo y } \\
\text { arcilla }\end{array}$ & $\begin{array}{l}\text { Limo y } \\
\text { arcilla }\end{array}$ & 46.4 & 36.8 & 31.1 & 29.3 & 28.7 \\
\hline
\end{tabular}

\section{Tabla 3}

Características reales de las muestras.

\begin{tabular}{|c|c|c|c|c|c|c|}
\hline \multicolumn{2}{|c|}{ DESCRIPCIÓN } & \multirow{2}{*}{$\begin{array}{r}\mathbf{5 1 \% A} \text {. } \\
\mathbf{4 9 \%} \text { T.C } \\
26.0\end{array}$} & \multirow{2}{*}{$\begin{array}{c}\text { 55\%A. } \\
\text { 45\% T.C } \\
23.00\end{array}$} & \multirow{2}{*}{$\begin{array}{c}\mathbf{6 0 \%} \mathbf{A} . \\
\mathbf{4 0 \%} \text { T.C } \\
21.0\end{array}$} & \multirow{2}{*}{$\begin{array}{c}\begin{array}{c}\mathbf{6 5 \%} \% \mathbf{A} \\
\mathbf{3 6} \% \text { T.C }\end{array} \\
19.0\end{array}$} & \multirow{2}{*}{$\begin{array}{c}\mathbf{7 0 \%} \% \mathbf{A} \\
\mathbf{3 0 \%} \text { T.C } \\
17.0\end{array}$} \\
\hline $\begin{array}{c}\text { Limite } \\
\text { Liquido }\end{array}$ & LL & & & & & \\
\hline $\begin{array}{l}\text { Limite } \\
\text { plástico }\end{array}$ & LP & 16.0 & 13.0 & 13.0 & 12.0 & 10.0 \\
\hline $\begin{array}{l}\text { Índice de } \\
\text { plasticidad }\end{array}$ & IP & 10.0 & 10.0 & 8.0 & 7.0 & 7.0 \\
\hline$\%$ Arena & $\% \mathrm{~A}$ & 53.3 & 63.0 & 66.4 & 69.99 & 70.4 \\
\hline AASHTO & AASHTO & A-4(2) & A-4(2) & A-2-4(0) & A-2-4(0) & A-2-4(0) \\
\hline SUCS & SUCS & $\mathrm{SC}$ & $\mathrm{SC}$ & $\mathrm{SC}$ & $\mathrm{SC}$ & $\mathrm{SC}$ \\
\hline
\end{tabular}

Tabla 4

Características reales de las muestras A-2-4(SC).

\begin{tabular}{|c|c|c|c|c|}
\hline Descripciór & $\begin{array}{c}\text { Rango } \\
\text { preferido }\end{array}$ & $\begin{array}{c}\text { 60\%A. } 40 \% \\
\text { T.C }\end{array}$ & $\begin{array}{c}\text { 65\%A. } \\
36 \% \text { T.C }\end{array}$ & $\begin{array}{c}\text { 70\%A. } \\
\text { 30\% T.C }\end{array}$ \\
\hline $\begin{array}{l}\text { Limite } \\
\text { Liquido }\end{array}$ & $30 \%-35 \%$ & 21.0 & 19.0 & 17.0 \\
\hline $\begin{array}{l}\text { Limite } \\
\text { plástico }\end{array}$ & $12 \%-22 \%$ & 13.0 & 12.0 & 10.0 \\
\hline $\begin{array}{l}\text { Índice de } \\
\text { plasticidad }\end{array}$ & - & 8.0 & 7.0 & 7.0 \\
\hline$\%$ Arena & - & 66.4 & 69.99 & 70.4 \\
\hline AASHTO & - & A-2-4(0) & A-2-4(0) & A-2-4(0) \\
\hline SUCS & - & $\mathrm{SC}$ & $\mathrm{SC}$ & $\mathrm{SC}$ \\
\hline
\end{tabular}


Tabla 5

Proctor intermedio con $60 \%$ A.C - 40\% T.C.

\begin{tabular}{ccccc}
\hline $\begin{array}{c}\text { Contenido } \\
\text { de humedad }\end{array}$ & 10.4 & 12.4 & 13.97 & 15.96 \\
\hline $\mathrm{MDS}\left(\mathrm{g} / \mathrm{cm}^{3}\right)$ & 1.652 & 1.847 & 1.868 & 1.803 \\
\hline
\end{tabular}

Tabla 6

Resultados de ensayos de albañilería.

\begin{tabular}{|c|c|c|c|c|c|c|}
\hline \multirow{2}{*}{ Descripción } & \multirow{2}{*}{\multicolumn{2}{|c|}{ Dimensiones }} & \multicolumn{4}{|c|}{ Resultados } \\
\hline & & & LTC 7\% & LTC $11 \%$ & LTC $15 \%$ & LTC 19\% \\
\hline \multirow{18}{*}{$\begin{array}{c}\text { Variación } \\
\text { Dimensional } \\
\text { de Unidades } \\
\text { de LTC }\end{array}$} & Largo & $\bar{x}$ & 250.18 & 250.18 & 250.30 & 250.13 \\
\hline & Ancho & $\bar{x}$ & 125.40 & 125.35 & 125.28 & 125.28 \\
\hline & Alto & $\bar{x}$ & 86.95 & 86.18 & 85.65 & 85.40 \\
\hline & Largo & Df & 250.00 & 250.00 & 250.00 & 250.00 \\
\hline & Ancho & Df & 125.00 & 125.00 & 125.00 & 125.00 \\
\hline & Alto & Df & 85.00 & 85.00 & 85.00 & 85.00 \\
\hline & Largo & $\sigma$ & 0.36 & 0.19 & 0.26 & 0.24 \\
\hline & Ancho & $\sigma$ & 0.21 & 0.16 & 0.16 & 0.19 \\
\hline & Alto & $\sigma$ & 0.34 & 0.23 & 0.21 & 0.22 \\
\hline & Largo & VDmm & -0.18 & -0.18 & -0.30 & -0.13 \\
\hline & Ancho & VDmm & -0.40 & -0.35 & -0.28 & -0.28 \\
\hline & Alto & VDmm & -1.95 & -1.18 & -0.65 & -0.40 \\
\hline & Largo & VD $\%$ & -0.07 & -0.07 & -0.12 & -0.05 \\
\hline & Ancho & VD $\%$ & -0.32 & -0.28 & -0.22 & -0.22 \\
\hline & Alto & $\mathrm{VD} \%$ & -2.29 & -1.39 & -0.76 & -0.47 \\
\hline & Largo & $\mathrm{CV} \%$ & 0.14 & 0.08 & 0.10 & 0.10 \\
\hline & Ancho & $\mathrm{CV} \%$ & 0.17 & 0.13 & 0.13 & 0.15 \\
\hline & Alto & $\mathrm{CV} \%$ & 0.39 & 0.27 & 0.25 & 0.26 \\
\hline \multirow{6}{*}{$\begin{array}{c}\text { Alabeo de } \\
\text { Unidades de } \\
\text { LTC }\end{array}$} & \multirow{3}{*}{ Convexidad } & $\bar{x}$ & 0.13 & 0.07 & 0.06 & 0.06 \\
\hline & & $\sigma$ & 0.02 & 0.01 & 0.00 & 0.00 \\
\hline & & $\mathrm{CV} \%$ & 15.38 & 14.29 & 0.00 & 0.00 \\
\hline & \multirow{3}{*}{ concavidad } & $\bar{x}$ & 0.30 & 0.19 & 0.18 & 0.12 \\
\hline & & $\sigma$ & 0.04 & 0.02 & 0.02 & 0.00 \\
\hline & & $\mathrm{CV} \%$ & 13.33 & 10.53 & 11.11 & 0.00 \\
\hline \multirow{3}{*}{$\begin{array}{l}\text { Succión de } \\
\text { las } \\
\text { Unidades de } \\
\text { LTC } \\
\end{array}$} & \multicolumn{2}{|c|}{$\bar{x}$} & 85.65 & 40.92 & 32.75 & 29.21 \\
\hline & \multicolumn{2}{|c|}{$\sigma$} & 6.69 & 4.03 & 2.50 & 2.58 \\
\hline & \multicolumn{2}{|c|}{$\mathrm{CV} \%$} & 7.81 & 9.85 & 7.63 & 8.83 \\
\hline \multirow{3}{*}{$\begin{array}{c}\text { Absorción } \\
\text { de las } \\
\text { Unidades de } \\
\text { LTC }\end{array}$} & \multicolumn{2}{|c|}{$\overline{\mathrm{x}}$} & 20.03 & 19.35 & 19.00 & 18.59 \\
\hline & \multicolumn{2}{|c|}{$\sigma$} & 1.19 & 0.91 & 0.91 & 0.79 \\
\hline & \multicolumn{2}{|c|}{$\mathrm{CV} \%$} & 5.94 & 4.70 & 4.79 & 4.25 \\
\hline \multirow{3}{*}{$\begin{array}{l}\text { Densidad de } \\
\text { Unidades de } \\
\text { LTC }\end{array}$} & \multicolumn{2}{|c|}{$\bar{x}$} & 1.49 & 1.50 & 1.51 & 1.52 \\
\hline & \multicolumn{2}{|c|}{$\sigma$} & 0.01 & 0.01 & 0.01 & 0.01 \\
\hline & \multicolumn{2}{|c|}{$\mathrm{CV} \%$} & 0.67 & 0.67 & 0.66 & 0.66 \\
\hline \multirow{3}{*}{$\begin{array}{l}\% \text { Vacios de } \\
\text { Unidades de } \\
\text { LTC }\end{array}$} & \multicolumn{2}{|c|}{$\bar{x}$} & 19.32 & 19.74 & 19.91 & 20.08 \\
\hline & \multicolumn{2}{|c|}{$\sigma$} & 0.12 & 0.13 & 0.14 & 0.10 \\
\hline & \multicolumn{2}{|c|}{$\mathrm{CV} \%$} & 0.62 & 0.66 & 0.70 & 0.50 \\
\hline \multirow{4}{*}{$\begin{array}{c}\text { R. } \\
\text { Compresión } \\
\text { de la } \\
\text { Unidad de } \\
\text { LTC (f'b) }\end{array}$} & \multicolumn{2}{|c|}{$\mathrm{fb}(\mathrm{kg} / \mathrm{cm} 2)$} & 21.28 & 30.32 & 44.45 & 55.48 \\
\hline & $\sigma$ & & 0.93 & 1.83 & 2.74 & 2.67 \\
\hline & $\mathrm{f}^{\prime} \mathrm{b}(\mathrm{kg}$ & & 20.35 & 28.49 & 41.71 & 52.81 \\
\hline & $\mathrm{CV}$ & & 4.37 & 6.04 & 6.16 & 4.81 \\
\hline
\end{tabular}




\begin{tabular}{|c|c|c|c|c|c|}
\hline \multirow{3}{*}{$\begin{array}{l}\text { R. } \\
\text { Compresión } \\
\text { de Pilas de } \\
\text { LTC (f'm) }\end{array}$} & fm $(\mathrm{kg} / \mathrm{cm} 2)$ & 16.31 & 18.08 & 21.81 & 29.17 \\
\hline & $\sigma$ & 0.66 & 0.73 & 1.02 & 1.54 \\
\hline & $\mathrm{f}^{\prime} \mathrm{m}(\mathrm{kg} / \mathrm{cm} 2)$ & 15.65 & 17.35 & 20.79 & 27.63 \\
\hline \multirow{3}{*}{$\begin{array}{c}\text { R. } \\
\text { Compresión } \\
\text { de Muretes } \\
\text { de LTC } \\
\left(V^{\prime} m\right)\end{array}$} & $\mathrm{Vm}(\mathrm{kg} / \mathrm{cm} 2)$ & 2.23 & 3.99 & 4.84 & 5.88 \\
\hline & $\sigma$ & 0.12 & 0.22 & 0.27 & 0.26 \\
\hline & $\mathrm{CV} \%$ & 5.38 & 5.51 & 5.58 & 4.42 \\
\hline
\end{tabular}

Tabla 7

Resultados del análisis de varianza y prueba estadística DMS.

\begin{tabular}{|c|c|c|c|c|}
\hline \multirow{2}{*}{ VARIABLES } & \multicolumn{3}{|c|}{ ANÁLISIS DE VARIANZA } & \multirow{2}{*}{ OBSERVACIONES } \\
\hline & Fc & Ft $5 \%$ & Ft $1 \%$ & \\
\hline $\mathrm{LP}(\mathrm{mm})$ & $\begin{array}{l}0.38 \\
\text { (nhds) }\end{array}$ & 3.24 & 5.29 & $\begin{array}{l}\text { Todos los lotes experimentales } 7 \%\left(\mathrm{~L}_{0}\right), 11 \% \\
\left(\mathrm{~L}_{1}\right), 15 \%\left(\mathrm{~L}_{2}\right) \text { y } 19 \%\left(\mathrm{~L}_{3}\right) \text { se comportan de la } \\
\text { misma manera. }\end{array}$ \\
\hline $\mathrm{AP}(\mathrm{mm})$ & $\begin{array}{l}0.58 \\
\text { (nhds) }\end{array}$ & 3.24 & 5.29 & $\begin{array}{l}\text { Todos los lotes experimentales } 7 \%\left(\mathrm{~L}_{0}\right), 11 \% \\
\left(\mathrm{~L}_{1}\right), 15 \%\left(\mathrm{~L}_{2}\right) \text { y } 19 \%\left(\mathrm{~L}_{3}\right) \text { se comportan de la } \\
\text { misma manera. }\end{array}$ \\
\hline \multirow{4}{*}{$\mathrm{HP}(\mathrm{mm})$} & \multirow{4}{*}{$\begin{array}{l}36.24 \\
\text { (das) }\end{array}$} & \multirow{4}{*}{3.24} & \multirow{4}{*}{5.29} & Mayor Comportamiento \\
\hline & & & & Lote experimental $19 \%\left(\mathrm{~L}_{3}\right)=85.404 \mathrm{~mm}$ \\
\hline & & & & Menor Comportamiento \\
\hline & & & & Lote experimental $7 \%\left(\mathrm{~L}_{0}\right)=86.952 \mathrm{~mm}$ \\
\hline \multirow{4}{*}{ CONVEX. } & \multirow{4}{*}{$\begin{array}{r}43.79 \\
(\text { das })\end{array}$} & \multirow{4}{*}{3.24} & \multirow{4}{*}{5.29} & Mayor Comportamiento \\
\hline & & & & $\begin{array}{l}\text { Lote experimental } 15 \%\left(\mathrm{~L}_{2}\right) \text { y } 19 \%\left(\mathrm{~L}_{3}\right) \text {, con } \\
0.062 \mathrm{~mm}\end{array}$ \\
\hline & & & & Menor Comportamiento \\
\hline & & & & Lote experimental $7 \%\left(\mathrm{~L}_{0}\right)=0.132 \mathrm{~mm}$ \\
\hline \multirow{4}{*}{ CONCAV. } & \multirow{4}{*}{$\begin{array}{l}54.30 \\
(\text { das })\end{array}$} & \multirow{4}{*}{3.24} & \multirow{4}{*}{5.29} & Mayor Comportamiento \\
\hline & & & & Lote experimental $19 \%\left(\mathrm{~L}_{3}\right)=0.122 \mathrm{~mm}$ \\
\hline & & & & Menor Comportamiento \\
\hline & & & & Lote experimental $7 \%\left(\mathrm{~L}_{0}\right)=0.296 \mathrm{~mm}$ \\
\hline \multirow{4}{*}{ SUCC. } & \multirow{4}{*}{$\begin{array}{l}184.97 \\
\text { (das) }\end{array}$} & \multirow{4}{*}{3.24} & \multirow{4}{*}{5.29} & Mayor Comportamiento \\
\hline & & & & Lote experimental $19 \%\left(\mathrm{~L}_{3}\right)=29.212 \%$ \\
\hline & & & & Menor Comportamiento \\
\hline & & & & Lote experimental $7 \%\left(\mathrm{~L}_{0}\right)=85.646 \%$ \\
\hline ABSOR. & $\begin{array}{l}2.02 \\
\text { (nhds) }\end{array}$ & 3.24 & 5.29 & $\begin{array}{l}\text { Todos los lotes experimentales } 7 \%\left(\mathrm{~L}_{0}\right), 11 \% \\
\left(\mathrm{~L}_{1}\right), 15 \%\left(\mathrm{~L}_{2}\right) \text { y } 19 \%\left(\mathrm{~L}_{3}\right) \text { se comportan de la } \\
\text { misma manera. }\end{array}$ \\
\hline \multirow{4}{*}{ DENS. } & \multirow{4}{*}{$\begin{array}{l}5.77 \\
(\text { das })\end{array}$} & \multirow{4}{*}{3.24} & \multirow{4}{*}{5.29} & Mayor Comportamiento \\
\hline & & & & Lote experimental $19 \%\left(\mathrm{~L}_{3}\right)=1.516 \mathrm{gr} / \mathrm{cm}^{3}$ \\
\hline & & & & Menor Comportamiento \\
\hline & & & & Lote experimental $7 \%\left(\mathrm{~L}_{0}\right)=1.492 \mathrm{gr} / \mathrm{cm}^{3}$ \\
\hline \multirow{4}{*}{ VACIOS } & \multirow{4}{*}{$\begin{array}{l}35.14 \\
\text { (das) }\end{array}$} & \multirow{4}{*}{3.24} & \multirow{4}{*}{5.29} & Mayor Comportamiento \\
\hline & & & & Lote experimental $19 \%\left(\mathrm{~L}_{3}\right)=20.084 \%$ \\
\hline & & & & Menor Comportamiento \\
\hline & & & & Lote experimental $7 \%\left(\mathrm{~L}_{0}\right)=19.324 \%$ \\
\hline \multirow{4}{*}{$\mathrm{fb}$} & \multirow{4}{*}{$\begin{array}{c}242.13 \\
\text { (das) }\end{array}$} & \multirow{4}{*}{3.24} & & Mayor Comportamiento \\
\hline & & & 520 & Lote experimental $19 \%\left(\mathrm{~L}_{3}\right)=55.478 \mathrm{~kg} / \mathrm{cm}^{2}$ \\
\hline & & & 3.29 & Menor Comportamiento \\
\hline & & & & Lote experimental $7 \%\left(\mathrm{~L}_{0}\right)=21.280 \mathrm{~kg} / \mathrm{cm}^{2}$ \\
\hline
\end{tabular}




\begin{tabular}{|c|c|c|c|c|}
\hline \multirow{4}{*}{$\mathrm{fm}$} & \multirow{4}{*}{$\begin{array}{c}148.40 \\
\text { (das) }\end{array}$} & \multirow{4}{*}{3.24} & \multirow{4}{*}{5.29} & Mayor Comportamiento \\
\hline & & & & Lote experimental $19 \%\left(\mathrm{~L}_{3}\right)=29.168 \mathrm{~kg} / \mathrm{cm}^{2}$ \\
\hline & & & & Menor Comportamiento \\
\hline & & & & Lote experimental $7 \%\left(\mathrm{~L}_{0}\right)=16.308 \mathrm{~kg} / \mathrm{cm}^{2}$ \\
\hline \multirow{4}{*}{$\mathrm{Vm}$} & \multirow{4}{*}{$\begin{array}{c}234.89 \\
\text { (das) }\end{array}$} & \multirow{4}{*}{3.24} & \multirow{4}{*}{5.29} & Mayor Comportamiento \\
\hline & & & & Lote experimental $19 \%\left(\mathrm{~L}_{3}\right)=5.884 \mathrm{~kg} / \mathrm{cm}^{2}$ \\
\hline & & & & Menor Comportamiento \\
\hline & & & & Lote exp erimental $7 \%\left(\mathrm{~L}_{0}\right)=2.228 \mathrm{~kg} / \mathrm{cm}^{2}$ \\
\hline
\end{tabular}

Detalle de fallas en unidad, pila y murete de LTC $19 \%$

Figura 1

Falla grieta vertical en unidades de LTC
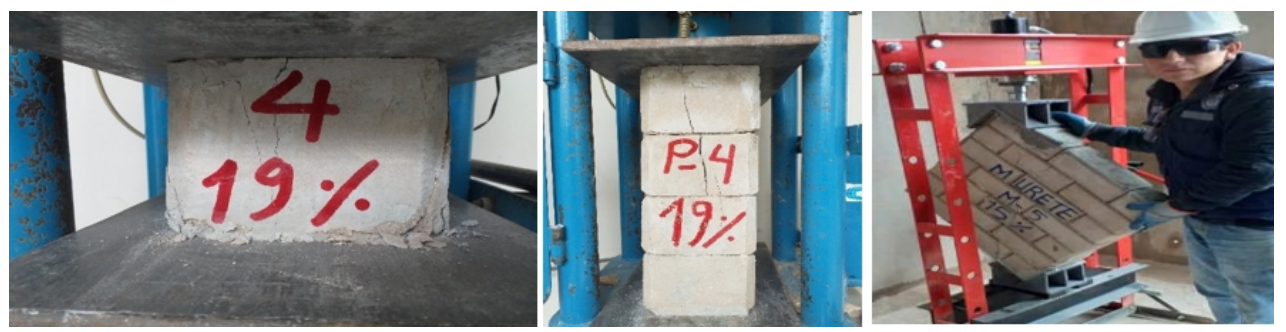

Tabla 8

Resumen de parámetros sísmicos

\section{PARAMETROS PARA MODELO SISMICO}

\begin{tabular}{|c|c|c|}
\hline Factor de zona & $(\mathrm{ZONA} 2), \mathrm{Z}=$ & 0.25 \\
\hline Perfil de suelo & Suelo Blando & S3 \\
\hline Factor deamplificaciórdel suelo & $\mathrm{S}=$ & 1.40 \\
\hline \multirow{2}{*}{ Periodos } & $\mathrm{T}_{\mathrm{P}}=$ & 1.00 \\
\hline & $\mathrm{T}_{\mathrm{L}}=$ & 1.60 \\
\hline Factor de uso & $\mathrm{U}=$ & 1.00 \\
\hline Coeficientebásicode reducción & $\mathrm{R}_{\mathrm{O}}=$ & 3.00 \\
\hline \multirow{2}{*}{ Irregularidad estructural en altura } & $\mathrm{I}_{\mathrm{AX}}=$ & 1.00 \\
\hline & $\mathrm{I}_{\mathrm{AY}}=$ & 1.00 \\
\hline \multirow{2}{*}{ Irregularidad estructural en planta } & $\mathrm{I}_{\mathrm{PX}}=$ & 1.00 \\
\hline & $\mathrm{I}_{\mathrm{PY}}=$ & 0.90 \\
\hline \multirow{2}{*}{ Coeficiente dđeducción } & $\mathrm{R}_{\mathrm{Xx}}=$ & 3.00 \\
\hline & $\mathrm{R}_{\mathrm{YY}}=$ & 2.70 \\
\hline Númerode pisos & $\mathrm{N}=$ & 1.00 \\
\hline
\end{tabular}


Tabla 9

Derivas de piso.

\begin{tabular}{cl}
\hline DIRECCIÓN & DERIVA \\
\hline$X$ & 0.004315 \\
\hline$Y$ & 0.00322 \\
\hline
\end{tabular}

\section{DISCUSIÓN}

Mediante el estudio realizado, se acepta la hipótesis planteada siendo admisible con derivas de $\mathrm{X}=0.004315, \mathrm{Y}=0.003262$, menores a 0.005 para albañilería, que indica el artículo 32 de la norma E.030-2018 del RNE, esto se logró con la adición de cemento al $19 \%$ en relación al peso de la mezcla, obteniendo LTC de Tipo I, con $52.81 \mathrm{~kg} / \mathrm{cm}^{2}$ de resistencia $\mathrm{f}^{\mathrm{b}} \mathrm{b}$

La presente investigación presenta diferencia con lo que sostiene (Abanto y Akarley, 2014), donde estudia la unidad de albañilería suelo cemento, pero con diferente material, con la cual al $20 \%$ de adición de cemento, llego a f'b de $74.78 \mathrm{~kg} / \mathrm{cm}^{2}$, lo cual con mi LTC $19 \%$ que tiene $\mathrm{f}^{\prime} \mathrm{b}$ de $52.81 \mathrm{~kg} / \mathrm{cm}^{2}$ difiere en un $29.34 \%$, es mucha la diferencia porque en porcentaje de adición de cemento hay $1 \%$ de diferencia, pero un factor primordial es las características del suelo está en un $75 \%$ de arena, y $25 \%$ de finos, es por ello que en el LTC 20\% hay mayor resistencia a la compresión, mientras que para la combinación $60 \%$ A. - $40 \%$ T., hay un $66.4 \%$ de arena y $33.1 \%$ de finos, reflejando la diferencia.

La presente investigación presenta diferencia con lo que sostiene (Meza, 2018), donde estudia la unidad de albañilería LTC, con características de suelo $48.60 \%$ de arena y $34.30 \%$ de limos y arcillas, con la cual al $7 \%, 11 \%, 15 \%$ y $20 \%$ de adición de cemento llego a f'b de 23.12; 27.99; 52.29; y $69.09 \mathrm{~kg} / \mathrm{cm}^{2}$ respectivamente; como vemos en mi investigación se tomó la combinación $60 \%$ A. - 40\% T., tengo un $66.4 \%$ de arena y $33.1 \%$ de finos, obteniendo un $\mathrm{f}^{\mathrm{b}} \mathrm{b}$ al $7 \%$. $11 \%, 15 \%$ y $19 \%$, de $20.35 ; 28.49,41.71$ y 52.81 $\mathrm{kg} / \mathrm{cm}^{2}$ respectivamente, donde se aprecia la diferencia en $11.98 \%, 1.76 \%, 20.23 \%$, y $23.56 \%$ respectivamente; lo que conlleva a decir que la investigación de Meza, 2018 se hizo con maquina hidráulica, mientras en mi caso lo hice con prensa manual, siendo las compactaciones muy diferentes.

En la Tabla 1, se presenta los resultados de los ensayos de mecánica de suelos, con respecto al contenido de arena se observa que la arena de cerro supera el rango óptimo de arenas que es de 55 al 75\%, lo cual tenemos como resultado $89 \%$, en el caso de la tierra colpar tenemos un $29.7 \%$ de arena, lo cual falta para llegar al porcentaje recomendado, es por ello que se planteó 5 mezclas: 51\% A.- 49\% T., 55\% A. $45 \%$ T., $60 \%$ A. - $40 \%$ T., $65 \%$ A. - $35 \%$ T., $70 \%$ A. $30 \% \mathrm{~T}$.
En la Tabla 2, Toirac Corral (2008), plantea que el óptimo porcentaje de arena es de $55 \%$ a $75 \%$, el porcentaje óptimo de limos es de $0 \%$ a $28 \%$, y el óptimo contenido de arcillas es de 15 a $18 \%$; de esto tenemos que las combinaciones: $55 \%$ A. - 45\% T., $60 \%$ A. - $40 \%$ T., $65 \%$ A. $-35 \%$ T., $70 \%$ A. $-30 \%$ T. están dentro del rango optimo establecido por Toirac Corral (2008).

En cuanto a los límites de consistencia IBCH(2009), plantea que el límite liquido tiene rango de tolerancia de $25 \%$ - $50 \%$, y rango preferido de $30 \%-35 \%$; en relación al límite plástico, el rango de tolerancia es de $10 \%$ - 25\%, el rango preferido es $12 \%-22 \%$; es por ello que en la Tabla 3, las combinaciones $60 \%$ A. $40 \%$ T., $65 \%$ A. - $35 \%$ T., $70 \%$ A. - 30\% T cumplen con el suelo optimo A-2-4 (SC) y el porcentaje óptimo de arena, conjuntamente analice con la Tabla 4 , lo cual descarte en función ala limite líquido y límite plástico, se optó por los rangos preferidos donde la combinación 70\% A. - 30\% T., queda descartado porque el límite plástico esta fuera del rango preferido, quedando solo dos combinaciones: $60 \%$ A. - $40 \%$ T., $65 \%$ A. - 35\% T., de estos, ninguno llega al rango preferido del límite líquido, pero se optó por elegir al que esté más cerca, y además con la finalidad de utilizar un poco más la materia prima (tierra colpar) del lugar se optó por la elección de la combinación: $60 \%$ A. - 40\% T; con esta combinación se realizó los ensayos respectivos en laboratorio de la DRTC-AMAZONAS.

En la Tabla 5, con los datos se considera la densidad máxima seca y la humedad óptima para esta investigación se optó por proctor intermedio con óptimo contenido de humedad de $13.3 \%$, y máxima densidad seca $1.874 \mathrm{gr} / \mathrm{cm}^{3}$.

En la Tabla 6, se presenta todos los resultados de los ensayos de albañilería realizados para cada lote experimental, observándose que la dosificación 19\% tiene mayor resistencia en $f^{\prime} b, f^{\prime} m, V^{\prime} m$.

En la Tabla 7, se presenta los Resultados del análisis de varianza y prueba estadística diferencia mínima significativa (DMS), para todos los lotes experimentales, de cada variable estudiada y de esto se deduce que, según el análisis estadístico, el que presentó mejor comportamiento es el lote experimental $\mathrm{L}_{3}(19 \%)$, lo cual para el modelamiento sísmico se hizo con unidades de albañilería LTC 19\%.

En cuanto a la Figura 1, de fallas de unidad, pila y murete observamos que la unidad tiene falla vertical considerándose como una falla ideal, además según Abanto y Poma(2014), en las pilas observamos una grieta vertical, producida por tracción debida a la expansión lateral, y para muretes se observa la falla diagonal cortando las unidades y mortero, lo cual de esto San Bartolomé lo designa como fallas ideales y por otro lado Rojas y Vidal (2014), consideran buena adherencia de unidades LTC con masa dun dun. 
En la Tabla 8, se presenta los parámetros para el modelamiento de vivienda para la Villa San Juan distrito de Magdalena, donde la zonificación sísmica establecida en Norma E 0.30-2018 del R.N.E., lo ubica en la zona sísmica 2 , con $Z=0.25$, además para el diseño estructural tenemos un perfil de suelo ( $\mathrm{S}$ $\left.=1.4, \mathrm{~T}_{\mathrm{P}}=1, \mathrm{~T}_{\mathrm{L}}=1.6\right)$, uso de la edificación $(\mathrm{U}=1)$, sistema sismorresistente $(\mathrm{Rxx}=3, \mathrm{Ryy}=2.70)$, siendo estas las consideraciones en el análisis sísmico.

En la Tabla 9, se presenta el resultando es derivas en $\mathrm{X}=0.004315$, en $\mathrm{Y}=0.003262$, manteniéndose menor al estipulado para albañilería que es 0.005 , además el modelo cumple las verificaciones de densidad de muros, esfuerzo máximo, control de fisuración, resistencia al corte, verificación de confinamiento, capacidad resistente, y diseño de vigas de amarre; con la cual se concluye que la respuesta es admisible dentro de su comportamiento sísmico, garantizando que la población se mantenga segura ante los efectos de un sismo, y también es necesario porque optimiza espacios en la distribución.

\section{CONCLUSIONES}

En relación con el modelamiento sísmico de una vivienda a base de unidades de suelo cemento, se determinó que es admisible la respuesta al modelo sísmico, llegando a tener derivas $(X=0.004315$, $\mathrm{Y}=0.003262)$, menores a 0.005 para albañilería, que indica el artículo 32 de la norma E.030-2018 del RNE.

La capacidad portante del suelo es $1.18 \mathrm{~kg} / \mathrm{cm}^{2}$, por lo tanto el suelo es blando y se caracteriza como suelo S3.

Elaboración de la unidad de albañilería de suelo cemento, con adición del 7\%, 11\%, 15\%, y 19\% de cemento respecto al peso de la mezcla (arena de cerro y tierra colpar) donde se determinó las propiedades físicas y mecánicas de las muestras de las mezclas de arena y tierra colpar concluyéndose que tiene un $66.4 \%$ de arena y $33.1 \%$ de finos y $0.6 \%$ de grava, además según AASHTO es de tipo A-2-4(0), asimismo según SUCS es SC. Elaborándose los LTC con dimensiones de fabricación de $25 \mathrm{~cm}$ de largo, $12.5 \mathrm{~cm}$ de ancho, y $8.5 \mathrm{~cm}$ de alto, de los cuales por prueba estadística de comparaciones múltiples Diferencia Mínima Significativa (DMS), se determinó que, para las propiedades físicas y mecánicas, el lote experimental que presentó mejor incidencia fue el $\mathrm{L}_{3}$ (19\%), que se utilizó en el modelo sísmico.

Las propiedades físicas y mecánicas de las muestras de las unidades de albañilería suelo cemento con adiciones al 7\%, 11\%, 15\% y 19\%, lote experimental $\mathrm{L}_{3}(19 \%)$ se comporta mejor, en relación a los demás lotes experimentales, obteniéndose las siguientes propiedades para el lote experimental 19\%: $\mathrm{f}^{\prime} \mathrm{b}=52.81$ $\mathrm{kg} / \mathrm{cm}^{2}, \mathrm{f}^{\prime} \mathrm{m}=27.63 \mathrm{~kg} / \mathrm{cm}^{2}, \mathrm{~V}^{\prime} \mathrm{m}=5.62 \mathrm{~kg} / \mathrm{cm}^{2}$.

El modelamiento en comparación con las normas E.070 y E030 del RNE, cumple con todo 1o especificado según dichas normas.

\section{REFERENCIAS BIBLIOGRÁFICAS}

Abanto Flores, P. J., \& Akarley Poma, L. M. (2014). Caracteristicas Fisicas y Mecanicas de Unidades de Albañileria Ecologicas Fabricadas con Suelo- Cemento en la Ciudad de Trujillo. Trujillo: Universidad Privada Antenor Orrego.

Flores Bravo, L. E., \& Moromi Nakata, I. (2006). Construir con Tierra Ayer y Hoy. Comporta miento de las Edificaciones con Tierra Cruda despues del Terremoto de Setiembre del 2005 Ciudad Lamas-Perú (págs. 67,68). IncihusaCricyt-Mendoza: Libro de edición argentina. Recuperado el 27 de octubre de 2017

Instituto Boliviano del Cemento Y el Hormigon. (2009). Manual Simplificado de Fabricacion de Bloques Prensados de Suelo Cemento. La Paz.

Linares Chavez, R. R. (2019). Estabilizacion de Suelos Arcillosos a Nivel de Subrasante con Adicion de Bolsas de Polietileno Fundido, Chachapoyas, 2018. Chachapoyas-Perú.

Meza Lopez, J. M. (2018). Evaluacion en Ensayos de Erosion Acelerada Aplicados a Ladrillos de Tierra Comprimida para la Construccion de Muros Perimetricos en Huancayo. Huancayo.

MTC. (2016). Ministerio de Transportes y Comunicaciones. Manual de ensayo de materiales. Perú.

Reglamento Nacional de Edificaciones. (s.f.). Noma Tecnica Peruana A.010- 2014. Diario el Peruano.

Reglamento Nacional de Edificaciones. (s.f.). Noma Tecnica Peruana A.020-2006. Diario el Peruano.

Reglamento Nacional de Edificaciones. (s.f.). Noma Tecnica Peruana E.020-2006. Diario el Peruano.

Reglamento Nacional de Edificaciones. (s.f.). Noma 
Tecnica Peruana E.030-2018. Diario el Peruano.

Reglamento Nacional de Edificaciones. (s.f.). Noma Tecnica Peruana E.070-2006. Diario el Peruano.

Salazar, C. (2018). Fundamentos básicos de la estadística. Primera edición. Perú.

San Bartolomé , A. (2005). Comentarios a la Norma Técnica de Edificación E.070 Albañileria.

Tejada Arias, A. M. (2013). Elaboracion de un Ladrillo Alternativo sin Coccion en Cajamarca. Cajamarca-Perú.

Toirac Corral, J. (2008). El Suelo Cemento como Material de Construccion. Ciencia y Sociedad, 53. 